\title{
La formation pratique des enseignants en Côte d'Ivoire
}

\section{Chronique internationale}

\section{Introduction}

La Côte d'Ivoire a placé depuis 1960 l'éducation au rang de priorité nationale et affiche la ferme volonté de scolariser la totalité des enfants du pays (Mian Bi, 2014). Ainsi, la loi dorientation $\mathrm{n}^{\circ}$ 95-696 du 7 septembre 1995 relative à l'enseignement stipule, dans son article premier, que "l'éducation est garantie à chaque citoyen...» (NeboutArkhurst et al., 2012, p. 21). La constitution du $1^{\text {er }}$ août 2000 consacre l'obligation pour l'État d'assurer un accès égal à l'éducation à tous les enfants du pays. Dans cette perspective, l'État a instauré la gratuité de l'éducation du CP1 (cours préparatoire $1^{\text {re }}$ année) à la classe de $6^{\text {e }}$. Et depuis 2015, un décret a été signé rendant la scolarité obligatoire de 6 à 16 ans.

L'article 3 de la loi d'orientation mentionnée stipule que le service public de l'enseignement est conçu et organisé en vue de permettre l'acquisition des savoirs (savoir-faire et savoir-être), des méthodes de travail et d'acquisition des connaissances, la formation de l'esprit critique et le développement de la sensibilité et de la curiosité.

Pour mettre en œuvre ce service public, l'État de la Côte d'Ivoire recrute et forme le personnel enseignant au niveau de chaque degré. La présente contribution, après une présentation sommaire du système éducatif, traite de la formation pratique des enseignants du préscolaire, du primaire et du secondaire en Côte d'Ivoire. 


\section{Méthodologie}

Pour atteindre les objectifs de la présente étude, nous avons fait une analyse documentaire. Cette analyse a été enrichie par des entrevues semi-dirigées organisées avec les responsables des différentes structures de formation que sont le CAFOP et l'ENS d'Abidjan. Dans le cadre de cette recherche, nous nous sommes inspiré des démarches proposées par Van der Maren (1995) en privilégiant une approche de type "analyse de contenu». Elle nous a permis de faire une description sommaire du système éducatif de la Côte d'Ivoire et de la formation pratique des enseignants.

\section{Description sommaire du système éducatif de la Côte d'Ivoire}

La Côte d'Ivoire est un pays de l'Afrique de l'Ouest, entourée au nord par le Burkina Faso et le Mali, à l'est par le Ghana et à l'ouest par le Liberia et la Guinée. Elle a une superficie de $322463 \mathrm{~km}^{2}$ et une population de 22671331 habitants, dont 49,3\% de femmes. Le système éducatif ivoirien est organisé en trois grands cycles (Sato, Manso, et Adiko, 2003; Mian Bi, 2014; Nebout-Arkhurst et al., 2012) : le primaire, le secondaire et le supérieur. Depuis le 22 novembre 2012, trois ministères sont responsables du secteur de l'éducation et de la formation : le ministère de l'Éducation nationale et de l'Enseignement technique (MENET) s'occupe des cycles primaire et secondaire; la formation professionnelle est sous la tutelle du ministère d'État, ministère de l'Emploi, des Affaires sociales et de la Formation professionnelle (MEMEASFP) ; le ministère de l'Enseignement supérieur et de la Recherche scientifique (MESRS) gère l'enseignement supérieur.

Pour ce qui est des descriptifs des différents cycles du système éducatif, il faut noter que le primaire comprend le préscolaire et l'élémentaire. Le niveau préscolaire couvre trois sections : la petite section, la moyenne section et la grande section. L'âge de la première inscription en petite section est de 3 ans. L'école élémentaire comprend six niveaux : le cours préparatoire $1^{\text {re }}$ et $2^{\mathrm{e}}$ année, le cours élémentaire $1^{\text {re }}$ et $2^{\mathrm{e}}$ année, le cours moyen $1^{\text {re }}$ et $2^{\mathrm{e}}$ année. Lâge de la première inscription au CP1 est de 6 ans. La fin du cycle primaire est sanctionnée par le Certificat d'études primaires et élémentaires (CEPE). L'âge d'entrée à l'école élémentaire est de 6 ans. Les statistiques de 2014 du MENET montrent que le taux brut de scolarisation au primaire est de 94,7 \%. Il est plus élevé chez les garçons (99,7\%) que chez les filles $(89,6 \%)$.

L'enseignement secondaire général et technique est subdivisé en deux cycles : le premier cycle et le second cycle. Le premier cycle part de la sixième $\left(6^{\mathrm{e}}\right)$ à la classe de troisième $\left(3^{\mathrm{e}}\right)$. La fin du premier cycle est sanctionnée par le diplôme du Brevet d'étude du premier cycle (BEPC). Depuis deux ans, l'entrée au premier cycle est conditionnée par l'admission au CEPE. Le second cycle, quant à lui commence de la classe de seconde $\left(2^{\mathrm{de}}\right)$ et prend fin à la classe de terminale. La fin du second cycle est sanctionnée par le diplôme du baccalauréat (BAC) organisé en séries : A, C, D pour l'enseignement général et $\mathrm{B}, \mathrm{E}, \mathrm{F}, \mathrm{G}$ pour l'enseignement technique. L'entrée au second cycle se fait sur orientation après la classe de $3^{\text {e }}$. Selon les statistiques de 2014 du MENET, le taux brut de scolarisation est de $50,8 \%$ au secondaire premier cycle et $27,2 \%$ au secondaire second cycle. Le taux d'achèvement du secondaire est de $34,6 \%$ au premier cycle et $21,8 \%$ au second cycle. 
La formation post-lycéenne débouche soit vers l'enseignement supérieur technique/professionnel, dans les grandes écoles, soit vers l'université (Sato et al., 2003; Mian Bi, 2014). Les grandes écoles publiques comme privées ont une vocation professionnelle, et les diplômes de technicien et d'ingénieur quelles offrent couvrent tous les domaines de l'activité commerciale, industrielle et agricole. Selon Sato et al. (2003), les grandes écoles publiques se répartissent en deux grandes catégories : celles dont la vocation est de former des ingénieurs et des techniciens supérieurs et celles dont la vocation est de former des formateurs (enseignants du primaire, du secondaire, etc.). Les universités aussi bien privées que publiques préparent aux diplômes d'études générales. Depuis 2012, l'enseignement supérieur ivoirien a intégralement basculé dans la réforme Licence-Master-Doctorat (LMD) avec l'adoption d'une nouvelle nomenclature des diplômes. Ainsi, selon Mian Bi (2014), dans l'enseignement supérieur ivoirien, on y prépare autant les diplômes universitaires (Licence, Master et Doctorat) que des diplômes professionnels (Ingénieur, Brevet de technicien, etc.).

\section{La formation pratique des enseignants}

La formation des enseignants du préscolaire et du primaire public est assurée par les 14 centres d'aptitude et de formation pédagogique (CAFOP). Pour le secondaire général public, les enseignants sont formés à l'École normale supérieure (ENS) d'Abidjan pour les disciplines autres que le sport, les arts plastiques et la musique. En effet, les enseignants de sport sont formés à l'Institut national de la jeunesse et des sports (INJS) et ceux d'arts plastiques et de musique à l'Institut national supérieur des arts et de l'action culturelle (INSAAC). En plus des enseignants du secondaire public, l'ENS d'Abidjan forme aussi les formateurs des CAFOP. Les formateurs de l'enseignement technique public sont formés à l'Institut pédagogique national de l'enseignement technique et professionnel (IPNETP). Au niveau institutionnel, les institutions de formation des enseignants dépendent de cinq ministères différents. En effet, l'ENS d'Abidjan dépend du MESRS; le CAFOP, du MENET; l'IPNETP, du MEMEASFP; l'INJS, du ministère de la Promotion de la jeunesse, des Sports et des Loisirs (MPJSL) et l'INSAAC de celui de la Culture et de la Francophonie. Dans l'enseignement supérieur public, les formateurs des universités publiques sont formés à l'Institut de recherche en pédagogie (IREP) de l'Université Félix Houphouët-Boigny de Cocody.

Dans le cadre du présent article, nous nous intéresserons à la formation pratique des enseignants du préscolaire, du primaire et du secondaire général.

\section{La formation des enseignants du préscolaire et du primaire}

Pour la formation des enseignants du préscolaire et du primaire, depuis 2013, l'admission se fait sur concours directs organisés par la Direction des examens et concours (DECO), avec le BAC toutes séries. Avant cette année, l'admission se faisait avec le BEPC pour la formation des instituteurs adjoints et avec le BAC pour la formation des instituteurs ordinaires. Ainsi, depuis l'année 2013, les CAFOP ne forment plus que des instituteurs ordinaires. La durée de formation est de deux années dont la première dans les CAFOP et la seconde en stage à responsabilité dans les écoles primaires publiques (EPP) d'application. La première année de formation au sein du CAFOP dure environ trente semaines et se déroule en deux périodes de formation théorique alternée par deux stages en tutelle de 21 jours chacun dans les EPP d'application (Nebout-Arkhurst et al., 2012). 
La formation théorique de type pluridisciplinaire (Nebout-Arkhurst et al., 2012) se fait dans cinq domaines disciplinaires pour un volume horaire annuel de 1248 heures :

- $\quad$ le domaine des langues est composé de la discipline du français;

- $\quad$ le domaine des sciences est composé des mathématiques, de la formation scientifique et des TICE;

- le domaine de l'univers social est composé de la psychopédagogie, de l'histoire-géographie, de la communication audiovisuelle $(\mathrm{CAV})$ et de l'éducation aux droits de l'Homme et à la citoyenneté (EDHC);

- le domaine des arts est composé de la discipline activité d'expression et de création (AEC);

- $\quad$ le dernier domaine, développement éducatif sportif et physique est composé de l'éducation physique et sportive (EPS) et des activités coopératives.

La formation théorique pluridisciplinaire est basée sur le renforcement des compétences disciplinaires, mais également sur la formation dans la didactique de la discipline.

Les stages en tutelle sont organisés dans les EPP d'application sous la responsabilité d'un maître d'application pour l'encadrement de la pratique pédagogique et d'un formateur disciplinaire de CAFOP. Avant la mise en stage de tutelle, la cellule de stage du CAFOP rencontre les maîtres d'application pour échanger avec eux sur le stage et recenser leurs besoins. Durant les 21 jours que dure le stage d'application, le formateur du CAFOP effectue des visites de classe. La première visite qui s'effectue une semaine après la prise en main de la classe par le stagiaire est une visite d'observation et la seconde, une visite d'évaluation. Les visites des formateurs sont des occasions de faire des remédiations sur la pratique pédagogique et aussi didactique. En effet, pendant ces visites, le formateur du CAFOP observe, à partir d'une grille préétablie, l'élève-maître pendant une séance de cours. Cette observation est suivie d'une séance d'autocritique de la part de l'élève-maître. Le maître d'application qui participe à cette séance propose aussi des remédiations. Le formateur du CAFOP, après une synthèse, apporte les éclairages et ajustements nécessaires. Pour l'organisation pratique de ces stages, le CAFOP dispose d'un cahier de suivi de tous les stagiaires. Chaque élève-maître dispose d'un cahier de critique qu'il doit renseigner chaque jour. Le maître d'application a aussi un cahier de suivi de la pratique quotidienne de l'élève-maître. Le formateur du CAFOP dispose, en plus du bulletin d'évaluation de la seconde visite, un imprimé qui résume les compétences liées à l'organisation, à l'animation et à l'évaluation du maître avec les remédiations. À la fin du stage, le maître d'application rédige un rapport qui porte sur la ponctualité, la personnalité et les activités pédagogiques du stagiaire.

Au cours de la première année de formation théorique, chaque formateur du CAFOP encadre deux ou trois élèves-maîtres pour la production d'un dossier pédagogique ou d'un document de projet éducatif. Le volume horaire alloué à cette activité est de 50 heures. La première année est sanctionnée par le Diplôme d'instituteur stagiaire (DIS). À la fin de cette année théorique, les notes de stages et de soutenance des dossiers pédagogiques sont transmises à la DECO tandis que les bulletins de visites sont transmis aux Inspections de l'enseignement primaire (IEP). 
La seconde année est consacrée au stage de responsabilité dans les EPP sous la direction conjointe de la Direction des écoles, lycées et collèges (DELC) du MENET et des IEP. Ce stage se termine par la titularisation faite par les IEP selon un calendrier dressé par la DECO.

L'une des forces de la formation pratique des enseignants du préscolaire et du primaire est la bonne coordination institutionnelle (Gervais et Desrosiers, 2005). En effet, toutes les structures qui interviennent appartiennent au même ministère, ce qui facilite l'organisation de la formation pratique des enseignants du préscolaire et du primaire. Cette formation intègre une bonne composante didactique et pédagogique et une bonne coordination entre le CAFOP et le milieu scolaire. Outre ces forces, faute d'équipement dans les CAFOP, cette formation intègre peu ou pas du tout les TIC malgré le fait qu'elles soient au programme comme discipline. Il faut ajouter à cela la réduction du temps de formation des instituteurs qui passe de trois à deux années.

\section{La formation des enseignants du secondaire}

Pour les métiers de l'éducation et de la formation, la formation des enseignants du secondaire général et des formateurs de CAFOP se déroule sur deux années à l'ENS d'Abidjan dans le domaine des sciences de l'éducation et de la formation selon la nomenclature du Conseil africain et malgache pour l'enseignement supérieur (CAMES). Si l'accès à la formation des enseignants se fait par concours directs ou par concours professionnels, celui des formateurs des CAFOP se fait exclusivement par concours professionnel. Les futurs enseignants des lycées et collèges issus des concours directs sont des étudiants diplômés des universités publiques dans les matières disciplinaires : mathématiques, français, langues (anglais, espagnol, allemand), sciences de la vie et de la Terre, sciences physiques et histoire-géographie. Les futurs enseignants des lycées et collèges issus des concours professionnels sont généralement des enseignants déjà en poste qui entrent à l'ENS d'Abidjan pour évoluer dans le métier. Quant aux futurs formateurs des CAFOP, ils sont instituteurs ordinaires. Depuis la généralisation de la réforme LMD dans l'enseignement supérieur ivoirien en 2012, l'ENS d'Abidjan forme au grade de Licence et Master dans le domaine des sciences de l'éducation.

Avec la nouvelle réforme des collèges en Côte d'Ivoire, les futurs enseignants du secondaire premier cycle entrent avec le niveau L2 ou Licence et préparent la Licence de professeur bivalent de collège. Ainsi, depuis la rentrée scolaire 2014-2015, la formation des professeurs bivalents du collège se fait dans cinq blocs :

- $\quad$ le bloc 1 est composé du français et de l'histoire-géographie;

- $\quad$ le bloc 2 est composé du français et de l'éducation aux droits de l'Homme et à la citoyenneté (EDHC);

- $\quad$ le bloc 3 est composé de l'anglais et l'EPS;

- $\quad$ le bloc 4 comprend les mathématiques et les TICE;

- $\quad$ le bloc 5 comprend les sciences physiques et les sciences de la vie et de la Terre (SVT). 
Les formateurs de CAFOP préparent la Licence de professeur de CAFOP dans différentes options en fonction des matières enseignées dans les CAFOP : mathématiques, formations scientifiques, psychopédagogiques, histoire-géographie et activité d'expression et de création (AEC). Les futurs enseignants du secondaire second cycle entrent avec le Master 1 et préparent un Master de professeur de lycée dans différentes options en fonction des disciplines enseignées au secondaire général: mathématiques, français, anglais, histoire-géographie, allemand, espagnol, SVT, sciences physiques et chimie, etc.

Contrairement à la formation des enseignants du primaire, la formation des enseignants du secondaire et des formateurs des CAFOP est une formation disciplinaire. Elle se déroule en quatre semestres dont les trois premiers au sein de l'ENS d'Abidjan pour une formation théorique et le dernier, consacré au stage de responsabilité dans les lycées, collèges et CAFOP de Côte d'Ivoire. Il faut noter qu'un semestre fait 60 crédits et qu'un crédit fait 25 heures selon la norme LMD appliquée à l'ENS d'Abidjan. Quelle que soit la discipline, le programme de formation théorique comprend des cours de renforcement disciplinaire et des cours de sciences de l'éducation (didactique, pédagogie générale, psychopédagogie, évaluation, philosophie de l'éducation, TICE, législation scolaire, etc.). Contrairement à l'année théorique de formation des enseignants du primaire, l'année théorique de formation à l'ENS d'Abidjan n'est pas ponctuée par des stages de tutelle. L'année de stage se fait sous la supervision d'un encadreur de l'ENS d'Abidjan et d'un professeur conseiller dans le lycée, collège ou CAFOP. L'encadreur de l'ENS est un enseignant disciplinaire de même que le professeur conseiller qui est désigné par l'administration de l'établissement. Avant la mise en stage, les étudiants suivent des séances de pré-stage. La séance de pré-stage débute par des renforcements didactiques qui portent sur des simulations de cours basé sur le programme en vigueur dans les établissements, lélaboration des fiches de planification pédagogique et l'utilisation des grilles d'évaluation d'une séance d'enseignement. À la fin de la séance de didactique débutent les microenseignements qui portent souvent sur un élément d'un cours normal et ont pour objectif par exemple la maîtrise d'une habileté précise ayant été présentée en cours de didactique : le questionnement, la variation des stimulus, l'utilisation du tableau, etc. Ces séances, avec des pairs jouant le rôle d'apprenants, sont enregistrées dans un studio d'enregistrement. À la fin des enregistrements, le formateur remet la cassette à l'étudiant pour faire son autoscopie à partir d'une grille déjà préétablie. Ensuite, le formateur et les étudiants passent à la phase d'exploitation dans la salle dédiée. Pendant cette séance d'exploitation, l'apprenant fait son autocritique, suivi des pairs qui font des critiques et observations et enfin le formateur fait la synthèse. À la phase de la remédiation, le formateur demande à un autre futur enseignant de présenter la même séquence de cours après avoir suivi la séance d'exploitation. En phase de microenseignement, tout le programme scolaire doit être vu. Et vers la fin du microenseignement, il est demandé aux futurs enseignants de faire des cours entiers, cette fois devant des élèves, avec ses pairs qui l'observent en utilisant une grille préétablie. Cette séance avec les élèves est filmée, suivie d'une autoscopie et une phase d'exploitation comme dans le microenseignement. À la fin du microenseignement, le futur enseignant est capable de tenir une classe de façon autonome.

Le pré-stage fini, les étudiants sont envoyés avec l'accord de la DELC du MENET dans leurs différents lieux de stage. Ce stage est un stage en responsabilité. En effet pendant ce stage, l'étudiant donne des cours, évalue les élèves et participe à toutes les activités pédagogiques au sein de l'établissement. Toutefois, ce stage en responsabilité se fait sous la supervision d'un formateur de l'ENS d'Abidjan et d'un professeur encadreur dans l'établissement. 
Avant le début de son stage, l'administration de l'ENS d'Abidjan remet au futur enseignant sa lettre de stage dans laquelle se trouvent des directives de stages à l'endroit de son professeur conseillé. L'étudiant lui-même ne dispose pas de documents lui donnant les directives à tenir pendant le stage pratique dans l'établissement. Toutes ces informations lui sont données pendant le pré-stage.

L'encadreur de l'ENS d'Abidjan effectue trois visites : les deux premières sont des visites de formation et la troisième est une visite d'évaluation. Lors des deux visites de formation, les futurs enseignants peuvent être observés pendant un enseignement ou pendant une séance de correction d'une évaluation. Chaque visite est suivie par une séance d'entretien qui se déroule exactement comme pendant le microenseignement. Et chaque visite est sanctionnée par un rapport rédigé par le professeur encadreur de l'ENS dans lequel il est mentionné le titre de la séance suivie, les observations et recommandations sur la visite. À la visite d'évaluation, le professeur conseiller devra rédiger un rapport de stage et attribuer une note de stage. Ce rapport de stage et la note sont déposés auprès de l'administration de l'ENS d'Abidjan.

Normalement, après la visite d'évaluation, le stage est terminé. Le futur enseignant rédige alors un mémoire de fin de formation qui est déposé auprès de l'administration de l'ENS. Mais comme le futur enseignant est en responsabilité, il reste à finir l'année scolaire avec le calcul des notes et le conseil de classe. C'est l'occasion pour lui de se familiariser avec ces activités pédagogiques de fin d'année scolaire. Toutefois, il est à noter qu'il ne participe pas aux examens nationaux en tant que stagiaire.

La titularisation qui est l'examen final se fait par un jury composé d'un inspecteur de l'enseignement secondaire de la Direction de la pédagogie et de la formation continue (DPFC) du MENET, de l'encadreur de l'ENS et d'un enseignant disciplinaire dans l'établissement, autre que le professeur conseiller.

La force de la formation pratique des enseignants du secondaire et des CAFOP est caractérisée par une concertation de plus en plus étroite entre universitaires de l'ENS d'Abidjan et professeurs conseillers (Nebout-Arkhurst et al., 2012), ce qui permet une meilleure cohérence de la formation. Par ailleurs, l'existence de laboratoires en sciences de l'éducation en particulier en didactique est un levier pour les innovations pédagogiques et la formation des formateurs d'enseignants. Il existe une coordination institutionnelle entre les services du MENET et ceux de l'ENS d'Abidjan quant à l'encadrement et au suivi des stagiaires.

Malgré ces points forts, il faut noter une insuffisance au niveau de l'encadrement des stagiaires sur le terrain. En effet, seulement le formateur disciplinaire de l'ENS d'Abidjan encadre le stagiaire sur le terrain. Pour un encadrement optimal, il serait important d'y associer un formateur des sciences de l'éducation. En plus de la question sur l'encadrement pendant le stage, il faut mentionner le manque de communication entre la DPFC et l'ENS d'Abidjan quant aux innovations pédagogiques en cours dans les établissements. Cette situation aboutit souvent à une inadéquation entre les programmes de formation à l'ENS d'Abidjan et les besoins réels des futurs enseignants sortants. En plus de ce dysfonctionnement, il faut noter la réduction du temps de formation des professeurs de CAFOP qui passe de trois à deux ans. 


\section{Conclusion}

Le présent article avait pour objectif de présenter la formation pratique des enseignants de la Côte d'Ivoire. Pour répondre à cet objectif, nous avons fait une analyse documentaire qui a été enrichie par des entrevues semi-dirigées des responsables de la formation à l'ENS d'Abidjan et des CAFOP. L'analyse des données montre que le cadre institutionnel de la formation de façon générale est une réelle force. De plus, la formation pratique des enseignants intègre une bonne dimension de sciences de l'éducation et une bonne organisation des stages dans les établissements d'enseignement. Pour une meilleure organisation, il serait indiqué de la mise en œuvre d'une étroite collaboration entre l'ENS d'Abidjan et la DPFC du MENET pour la prise en compte des innovations pédagogiques dans les programmes de formation à l'ENS d'Abidjan. Et pour améliorer la qualité de formation, il est souhaitable de mettre en place une plateforme de formation en ligne dans le domaine de l'intégration des TIC et des sciences de l'éducation de façon générale.

\section{Références}

Gervais, C et Desrosiers, P. (2005). L'école, lieu de formation d'enseignants. Questions et repères pour l'accompagnement de stagiaires. Québec, QC : Presses de l'Université de Laval.

Mian Bi, S. A. (2014). Côte d'Ivoire. Intégration des TIC aux systèmes d'éducation et de formation en Afrique. Dans Association pour le développement de l'éducation en Afrique (dir.), Intégration des TIC dans les systèmes d'éducation et de formation en Afrique : Expériences de l'Argentine, du Burkina Faso, de la Côte d'Ivoire, du Paraguay, du Sénégal, de la Tunisie et de l'Uruguay (p. 89-128). Repéré à http://www.adeanet.org/portalv2/sites/default/files/etudes_web fr.pdf

Nebout-Arkhurst, P., M’Boua, P. A., Atta, G. K. Y., Kouame, P. K., Likpa, S. H. et Kouakou, F. G. (2012). La formation des enseignants en Côte d'Ivoire. Dans Commission internationale de l'enseignement mathématique (dir.), La formation des enseignants en Afrique francophone subsabarienne. Cinq études de cas : Burkina Faso, Côte d'Ivoire, Mali, Niger, Sénégal (p. 18-30). Repéré à http://www.mathunion.org/fileadmin/ICMI/docs/Rapport final. 10 2012_website.pdf

Sato, A., Manso, L. et Adiko, A. (2003). Inventaire de l'enseignement supérieur en Côte d'Ivoire. Repéré à http://www.ide.go.jp/English/Publish/Download/Ars/08.html

Van der Maren, J.-M. (1995). Méthodes de recherche pour l'éducation. Montréal, QC : Presses de l'Université de Montréal. 